\title{
Retraction
}

\section{Retracted: Modeling, Real-Time Estimation, and Identification of UWB Indoor Wireless Channels}

\author{
International Journal of Antennas and Propagation
}

Received 7 March 2017; Accepted 7 March 2017; Published 13 July 2017

Copyright (C) 2017 International Journal of Antennas and Propagation. This is an open access article distributed under the Creative Commons Attribution License, which permits unrestricted use, distribution, and reproduction in any medium, provided the original work is properly cited.

International Journal of Antennas and Propagation has retracted the article titled "Modeling, Real-Time Estimation, and Identification of UWB Indoor Wireless Channels" [1]. The article was found to contain a substantial amount of material from the following published article: Yanyan Li, Mohammed Olama, Seddik Djouadi, Aly Fathy, Teja Kuruganti: "Stochastic UWB wireless channel modeling and estimation from received signal measurements." Radio and Wireless Symposium, pp. 195-198. IEEE, 2009.

The reuse includes most of the introduction, some of the methods and results, and all the conclusion. The authors do not agree with this retraction as Li et al. were cited as "Some preliminary results using SDEs to model UWB channels were presented initially in [19]."

The authors believe that the approximation of the timevarying wireless channel impulse response is more general and harder to prove and solve (providing the arbitrary order approximation while Li et al. provided the fixed order approximation). The proof of Theorem 2 is different and required additional steps. New stochastic models for indoor wireless channels and their statistics are provided (eq. (26)-(28)), as well as new stochastic models for UWB indoor wireless channels and their statistics (eq. (30)-(32)) and generalized forms of the models in Li et al.'s study. The received signal $y(t)$ has triple summations and a phase term, while the received signal in Li et al.s study (eq. (14)) has only double summations without a phase term.

\section{References}

[1] M. M. Olama, S. M. Djouadi, Y. Li, and A. Fathy, "Modeling, real-time estimation, and identification of UWB indoor wireless channels," International Journal of Antennas and Propagation, vol. 2013, Article ID 467670, 8 pages, 2013. 

UWB Indoor Wireless Channels

\author{
Mohammed M. Olama, ${ }^{1}$ Seddik M. Djouadi, ${ }^{2}$ Yanyan Li, ${ }^{2}$ and Aly Fathy ${ }^{2}$ \\ ${ }^{1}$ Computational Sciences \& Engineering Division, Oak Ridge National Laboratory, P.O. Box 2008, MS 6085, \\ Oak Ridge, TN 37831, USA \\ ${ }^{2}$ Electrical Engineering \& Computer Science Department, University of Tennessee, 1520 Middle Drive, Knoxville, TN 37996, USA
}

Correspondence should be addressed to Mohammed M. Olama; olamahussemm@ornl.gov

Received 12 July 2013; Accepted 1 November 2013

Academic Editor: Ai Bo

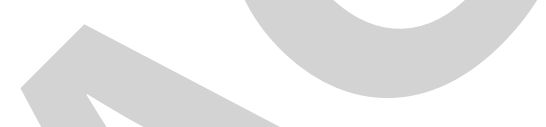

Copyright (C) 2013 Mohammed M. Olama et al. This is an open access article distributed under the Creative Commons Attribution License, which permits unrestricted use, distribution, and reproduction in any medium, provided the original work is properly cited.

Stochastic differential equations (SDEs) are used to model ultrawideband (UWB) indoor wireless channels. We show that the impulse responses for time-varying indoor wireless channels can be approximated in a mean-square sense as close as desired by impulse responses that can be realized by SDEs. The state variables represent the inphase and quadrature components of the UWB channel. The expected maximization and extended Kalman filter are employed to recursively identify and estimate the channel parameters and states, respectively, from online received signal strength measured data. Both resolvable and nonresolvable multipath received signals are considered and represented as small-scaled Nakagami fading. The proposed models together with the estimation algorithm are tested using UWB indoor measurement data demonstrating the method's viability and the results are presented.

\section{Introduction}

Ultrawideband (UWB) communication systems have recently attracted significant interest from both the research community and industry since the Federal Communications Commission (FCC) allowed limited unlicensed operation of UWB devices in the USA [1]. They are commonly defined as systems that have either more than $20 \%$ relative bandwidth or more than $500 \mathrm{MHz}$ absolute bandwidth. UWB technology has many benefits, including high data rate, low interference, less sensitivity to multipath fading, low transmit power, and availability of low cost transceivers [2]. Industrial standards such as IEEE 802.15.3a and 802.15.4a have been established in recognizing these developments.

The ultimate performance limits of a communication system are determined by the channel it operates in [3]. Realistic channel models are thus of utmost importance for system design and testing. UWB propagation channels show fundamental differences from conventional (narrowband) ones in many respects $[4,5]$, and therefore the established (narrowband) channel models cannot be used. A number of UWB channel models have been proposed in the literature.
A model for frequency range below $1 \mathrm{GHz}$ is suggested in [6]. A statistical model that is valid for a frequency range from 3 to $10 \mathrm{GHz}$ is proposed in [7] and is accepted by the IEEE 802.15.4a task group as a standard model for evaluation of UWB system proposals. Significant experimental work in office, residential, and industrial environments has been reported in this field such as in $[8,9]$. Most of the proposed channel models are based on characterizing the discrete multipath components. Although these models are able to capture the statistics of the channel, they cannot be specified by a finite number of parameters since their impulse responses are general functions of time and space and therefore are not easy to estimate directly from measurements.

A necessary and sufficient condition for representing any time-varying (TV) impulse response (IR) in stochastic state-space form is that it is factorizable into the product of two separate functions of time and space [10]. However, in general this is not the case for the IR of wireless channels. We show that the IR of indoor wireless channels can be approximated in the mean-square sense as close as desired by factorizable impulse responses that can be realized by stochastic differential equations (SDEs) in state-space form. 
In particular, the SDEs are used to model UWB indoor channels and are combined with system identification algorithms to extract various parameters of the channel from received signal measurement data. The expected maximization (EM) and the extended Kalman filter (EKF) are employed in estimating channel parameters as well as the inphase and quadrature components, respectively. The EM and EKF are chosen since they are recursive and therefore can be implemented online. These algorithms have been recently utilized in [11-13] to estimate the channel parameters and states in narrowband environments, and therefore the formulations of these algorithms are not presented in this paper. Experiments are conducted in our UWB laboratory to collect received signal strength measured data, which are used to determine the applicability of the proposed models. These models can be used in the development of a practical channel simulator that replicates wireless channel characteristics and produces outputs that vary in a similar manner to the variations encountered in a real-world UWB channel environment.

Recently, there have been several papers on the application of SDEs to modeling propagation phenomena in radar scattering and wireless communications. SDEs have been successfully used to analyze $K$-distributed noise in electromagnetic scattering in [14]. Autoregressive stochastic models for the computer simulation of correlated Rayleigh fading processes are investigated in [15]. A first-order stochastic autoregressive model for a flat stationary wireless channel is introduced in [16]. Stochastic channel models based on SDEs for cellular and ad hoc networks have been presented in [12, $17,18]$. Some preliminary results using SDEs to model UWB channels were presented initially in [19]. The advantage of using SDE methods is based on the computational simplicity of the algorithm simply because estimation is done recursively. This means that there is no need to store and process all measurements; rather, at each time step, the estimator is updated using the previous estimator values and the new innovations.

The paper is organized as follows. In Section 2, the general TV narrowband and UWB indoor wireless channel impulse responses are introduced. In Section 3, we show that the impulse responses for TV indoor wireless channels can be approximated in a mean-square sense as close as desired by impulse responses that can be realized by SDEs. The stochastic UWB channel models are developed in Section 4. In Section 5, experimental setup and numerical results are presented. Finally, Section 6 provides concluding remarks.

\section{The General Time-Varying Impulse Response for Indoor Wireless Channels}

The general TV impulse response (in complex baseband) of an indoor wireless fading channel is typically represented by Saleh-Valenzuela (SV) model given as [20]

$$
\begin{array}{r}
C(t ; \tau)=\sum_{l=1}^{L} \sum_{k=1}^{K_{l}} a_{k l}(t, \tau) \exp \left(j \phi_{k l}(t, \tau)\right) \\
\times \delta\left(t-T_{l}(t)-\tau_{k l}(t)\right),
\end{array}
$$

where $C(t ; \tau)$ is the impulse response of the channel at time $t$, due to an impulse applied at time $t-\tau, a_{k l}(t, \tau)$ and $\phi_{k l}(t, \tau)$ are, respectively, the random TV tap weight and phase of the $k$ th component in the $l$ th cluster, $T_{l}(t)$ is the delay of the $l$ th cluster, $\tau_{k l}(t)$ is the delay of the $k$ th multipath component (MPC) relative to the $l$ th cluster arrival $T_{l}(t), \delta(\cdot)$ is the Dirac delta function, $K_{l}$ is the total number of MPCs within the $l$ th cluster, and $L$ is the total number of clusters that can either be assumed fixed [21] or considered to be a random variable [7]. Let $s(t)$ be the transmitted signal; the received signal is then given by

$$
\begin{aligned}
y(t)=\sum_{l=1}^{L} \sum_{k=1}^{K_{l}} & a_{k l}(t, \tau) \exp \left(j \phi_{k l}(t, \tau)\right) \\
& \times s\left(t-T_{l}(t)-\tau_{k l}(t)\right)+v(t),
\end{aligned}
$$

where $v(t)$ is the measurement noise process.

For narrowband systems, complex Gaussian fading is conventionally used to describe the small-scale fading. More precisely, the equivalent complex baseband representation consists of Rayleigh-distributed amplitude and uniformly distributed phase. This can be related theoretically to the fact that a large number of multipath components fall into each resolvable delay bin, so that the central limit theorem is valid [3]. Therefore, $a_{k l}(t, \tau)$ and $\phi_{k l}(t, \tau)$ are statistically independent Rayleigh and uniform (over $[0,2 \pi]$ ) random processes, respectively [20].

In UWB systems, the central limit theorem is not valid, and a number of alternative amplitude distributions have been proposed in the literature. The most common empirically determined amplitude distribution in many UWB environments is Nakagami distribution, which is observed in $[6,8]$ and considered in the IEEE 802.15.4a standard [7]. Therefore, in UWB systems, $a_{k l}(t, \tau)$ and $\phi_{k l}(t, \tau)$ are statistically independent Nakagami and uniform random processes, respectively. In the next section, the corresponding impulse response with $a_{k l}(t, \tau)$ and $\phi_{k l}(t, \tau)$ for the timevarying indoor wireless channels in (1) is approximated in a mean-square sense as close as desired by SDEs.

\section{Approximating the Time-Varying Impulse Response for Indoor Wireless Channels by SDEs}

Now, we want to represent the TV IR in (1) with a stochastic state-space form in order to allow well-developed tools of estimation and identification to be applied to this class of problems. The following theorem states a necessary and sufficient condition for the realization of the TV IR.

Theorem 1 (see [10]). The impulse response $C(t ; \tau)$ of a TV system has a stochastic state-space realization if and only if it is factorizable; that is, there exist functions $g(\cdot)$ and $f(\cdot)$ such that for all $t$ and $\tau$, one has

$$
C(t ; \tau)=g(t) f(\tau) .
$$

It is readily seen from the expression of the IR $C(t ; \tau)$ of the indoor wireless channels in (1) that in general it is not 
factorizable in the form (3) since $a_{k l}(t, \tau)$ and $\phi_{k l}(t, \tau)$ are arbitrary functions of $t$ and $\tau$. However, one will show that in general $C(t ; \tau)$ can be approximated as close as desired by a factorizable IR function.

Theorem 2. In general, the IR $C(t ; \tau)$ of the indoor wireless channel in (1) can be approximated as close as desired by a factorizable function.

Proof. The IR $C(t ; \tau)$ of the indoor wireless channel has finite energy; that is,

$$
C(t ; \tau) \in L^{2}([0, \infty) \times[0, \infty)),
$$

where $L^{2}([0, \infty) \times[0, \infty))$ is the Hilbert space of square integrable complex valued functions defined on $[0, \infty) \times$ $[0, \infty)$ with the norm

$$
\begin{array}{r}
\|f\|_{2}^{2}:=\iint_{[0, \infty)[0, \infty)}|f(t ; \tau)|^{2} d \tau d t<\infty, \\
\quad f(t ; \tau) \in L^{2}([0, \infty) \times[0, \infty)) .
\end{array}
$$

Likewise define $L^{2}([0, \infty))$ as the standard Hilbert space of square integrable complex valued functions defined on $[0, \infty)$ under the norm

$$
\|x\|_{2}^{2}:=\int_{0}^{\infty}|x(t)|^{2} d t, \quad x \in L^{2}([0, \infty)) .
$$

The space $L^{2}([0, \infty))$ contains all finite energy signals defined on $[0, \infty)$. The IR $C(t ; \tau)$ of the channel has a finite energy and belongs to $L^{2}([0, \infty) \times[0, \infty))$; that is,

$$
\|C\|_{2}^{2}:=\iint_{[0, \infty)[0, \infty)}|C(t ; \tau)|^{2} d \tau d t<\infty
$$

Since the transmitted and received signals are finite energy signals, the IR can be viewed as an integral operator mapping transmitted signals in $L^{2}([0, \infty))$ into $L^{2}([0, \infty))$; that is, if $s \in L^{2}([0, \infty))$, then

$$
\begin{aligned}
& \qquad \begin{array}{r}
y(t)=\int_{0}^{\infty} C(t ; \tau) s(t-\tau) d \tau \in L^{2}([0, \infty)) . \\
\text { The tensor space } \\
L^{2}([0, \infty)) \otimes L^{2}([0, \infty)) \\
:=\left\{F(t, \tau) \in L^{2}([0, \infty) \times[0, \infty)):\right. \\
F(t, \tau)=\sum_{k=1}^{n} \alpha_{k}(t) \phi_{k}(\tau), \\
\alpha_{k}(t) \in L^{2}([0, \infty)), \\
\left.\phi_{k}(\tau) \in L^{2}([0, \infty)), \forall n \text { integer }\right\}
\end{array}
\end{aligned}
$$

is dense in $L^{2}([0, \infty) \times[0, \infty))$; that is, for any $\varepsilon>0$, there exist $\left\{\alpha_{k}\right\}_{1}^{n},\left\{\phi_{k}\right\}_{1}^{n} \subset L^{2}([0, \infty))$ such that $[22]$

$$
\mu_{n}:=\left\|C(t ; \tau)-\sum_{k=1}^{n} \alpha_{k}(t) \phi_{k}(\tau)\right\|_{2} \leq \varepsilon
$$

and $\mu_{n_{n} \rightarrow \infty} 0$. This implies that in the $\|\cdot\|_{2}$-norm, $C(t ; \tau)$ can be approximated to any desired accuracy by an IR of the form $\sum_{k=1}^{n} \alpha_{k}(t) \phi_{k}(\tau)$, which is factorizable by putting $g(t):=\left[\begin{array}{llll}\alpha_{1}(t) & \alpha_{2}(t) & \cdots & \alpha_{n}(t)\end{array}\right]$ and $f(\tau):=$ $\left[\begin{array}{llll}\phi_{1}(\tau) & \phi_{2}(\tau) & \cdots & \phi_{n}(\tau)\end{array}\right]^{T}$, where $T$ denotes vector or matrix transpose.

The optimal approximation of $C(t ; \tau)$ by functions in $L^{2}([0, \infty)) \otimes L^{2}([0, \infty))$ corresponding to $(10)$ can be written as

$$
\mu_{n}^{*}:=\inf _{\substack{\alpha_{k}(t) \in L^{2}([0, \infty)), \phi_{k}(\tau) \in L^{2}([0, \infty))}}\left\|C(t ; \tau)-\sum_{k=1}^{n} \alpha_{k}(t) \phi_{k}(\tau)\right\|_{2} .
$$

For arbitrary $n$, expression (11) is nothing but the shortest distance between the impulse function $C(t ; \tau)$ and the space $L^{2}([0, \infty)) \otimes L^{2}([0, \infty))$; that is, $\mu_{n}^{*}=\operatorname{dist}\left(C(t ; \tau), L^{2}([0, \infty)) \otimes\right.$ $\left.L^{2}([0, \infty))\right)$. The problem is to find the minimizing functions $\left\{\alpha_{k}\right\}_{1}^{n},\left\{\phi_{k}\right\}_{1}^{n}$. This problem has been solved in [22] for arbitrary positive $n$. However, for fixed positive $n$, the problem becomes the shortest distance, denoted by $\mu_{n}^{o}:=$ $\operatorname{dist}(C(t ; \tau), S)$, from $C(t ; \tau)$ to the set

$$
\begin{gathered}
S:=\left\{F(t, \tau)=\sum_{k=1}^{n} \alpha_{k}(t) \phi_{k}(\tau), \alpha_{k}(t) \in L^{2}([0, \infty)),\right. \\
\left.\phi_{k}(\tau) \in L^{2}([0, \infty)), n>0 \text { fixed }\right\} .
\end{gathered}
$$

Note that $S$ is not a subspace and is not a convex set since it is not closed under addition. Therefore, the argument presented in [22] does not hold anymore since the orthogonal projection onto the set $S$ is not linear. Following [22], the impulse response is viewed as an integral operator $T$ mapping transmitted signals from $L^{2}([0, \infty))$ into $L^{2}([0, \infty))$; that is, if $s_{l} \in$ $L^{2}([0, \infty))$, then $y_{l}(t) \in L^{2}([0, \infty))$, where

$$
y_{l}(t)=\left(T s_{l}\right)(t):=\int_{0}^{\infty} C(t ; \tau) s_{l}(t-\tau) d \tau .
$$

Since the impulse response is finite energy, the operator $T$ is a Hilbert-Schmidt or a trace class 2 operator $[22,23]$. Let us denote the class of Hilbert-Schmidt operators acting from $L^{2}([0, \infty))$ into $L^{2}([0, \infty))$ by $C_{2}$ and the HilbertSchmidt norm $\|\cdot\|_{\mathrm{HS}}$ is defined by

$$
\|T\|_{\mathrm{HS}}=\sqrt{\iint_{[0, \infty)[0, \infty)}|C(t ; \tau)|^{2} d \tau d t}, \quad T \in C_{2} .
$$

The operator $T$ admits a spectral factorization of the form $[22,23]$

$$
T=\sum_{i=1}^{\infty} \lambda_{i} \nu_{i} \otimes \psi_{i}
$$

where $\otimes$ is the tensor product, $\lambda_{i}>0$ with $\lambda_{i} \geq$ $\lambda_{i+1}, i=1,2, \ldots$, and both $\left\{v_{i}\right\}_{1}^{\infty}$ and $\left\{\psi_{i}\right\}_{i=1}^{\infty}$ are orthonormal sequences in $L^{2}([0, \infty))$ and are given by

$$
\nu_{i}(t)=\int_{0}^{\infty} C(t ; \tau) \psi_{i}(\tau) d \tau, \quad \psi_{i}(\tau)=\int_{0}^{\infty} C(t ; \tau) \nu_{i}(t) d t .
$$


The sum (15) has either a finite or countably infinite number of terms. The above representation is unique. The HilbertSchmidt norm of $T$ is also given by

$$
\|T\|_{\mathrm{HS}}=\sqrt{\sum_{i} \lambda_{i}^{2}}<\infty .
$$

The spectral factorization (15) yields the following representation for the impulse response $C(t ; \tau)[23]$ :

$$
C(t ; \tau)=\sum_{i=1}^{\infty} \lambda_{i} \nu_{i}(t) \psi_{i}(\tau)
$$

It follows that the minimum in (11) is given by taking.

$$
\alpha_{k}(t)=\lambda_{k} \nu_{k}(t), \quad \phi_{k}(\tau)=\psi_{k}(\tau), \quad k=1,2, \ldots, n .
$$

To further illustrate this result, note that $\left\{v_{i}\right\}_{i=1}^{\infty}$ and $\left\{\psi_{i}\right\}_{i=1}^{\infty}$ are orthonormal systems, and simple computations yield

$$
\left\|C(t ; \tau)-\sum_{k=1}^{n} \lambda_{k} \nu_{k}(t) \psi_{k}(\tau)\right\|_{2}^{2}=\|C(t ; \tau)\|_{2}^{2}-\sum_{k=1}^{n} \lambda_{k}^{2} .
$$

Schmidt in [24] showed that for any other functions $f_{i}(t), g_{i}(\tau) \in L^{2}([0, \infty)), i=1,2, \ldots, n$, the following inequality holds:

$$
\left\|C(t ; \tau)-\sum_{k=1}^{n} f_{k}(t) g_{k}(\tau)\right\|_{2}^{2} \geq\|C(t ; \tau)\|_{2}^{2}-\sum_{k=1}^{n} \lambda_{k}^{2},
$$

and therefore the minimum in (11) is given by (19). The optimal approximation follows as

$$
\mu_{n}^{o}=\left\|C(t ; \tau)-\sum_{k=1}^{n} \lambda_{k} \nu_{k}(t) \psi_{k}(\tau)\right\|_{2}=\sqrt{\sum_{k=n+1}^{\infty} \lambda_{k}^{2}}
$$

and $\mu_{n}^{o} \underset{n \rightarrow \infty}{\rightarrow} 0$. That is, by increasing $n$, the RHS of (22) can be made arbitrarily small. In other words, for large enough $n$, the following approximation is optimal in a mean-square sense:

$$
C(t ; \tau) \approx \sum_{i=1}^{n} \lambda_{i} \nu_{i}(t) \psi_{i}(\tau)
$$

and is factorizable.

The corresponding SDE is then given by [10]

$$
d X(t)=f(t) d W(t), \quad y(t)=g(t) X(t),
$$

where $X(t)$ is the state of the channel and $W(t)$ is the standard Brownian motion. Since state-space realizations of impulse responses are not unique [10], a realization of the following form in terms of the inphase and quadrature components for the $k$ th path within the $l$ th cluster can be used as $[12,17]$

$$
\begin{aligned}
d X_{k l}^{I}(t) & =A_{k l}^{I}(t) X_{k l}^{I}(t) d t+B_{k l}^{I}(t) d W_{k l}^{I}(t), \\
I_{k l}(t) & =H_{k l}^{I}(t) X_{k l}^{I}(t), \\
d X_{k l}^{\mathrm{Q}}(t) & =A_{k l}^{\mathrm{Q}}(t) X_{k l}^{\mathrm{Q}}(t) d t+B_{k l}^{\mathrm{Q}}(t) d W_{k l}^{\mathrm{Q}}(t), \\
Q_{k l}(t) & =H_{k l}^{\mathrm{Q}}(t) X_{k l}^{\mathrm{Q}}(t),
\end{aligned}
$$

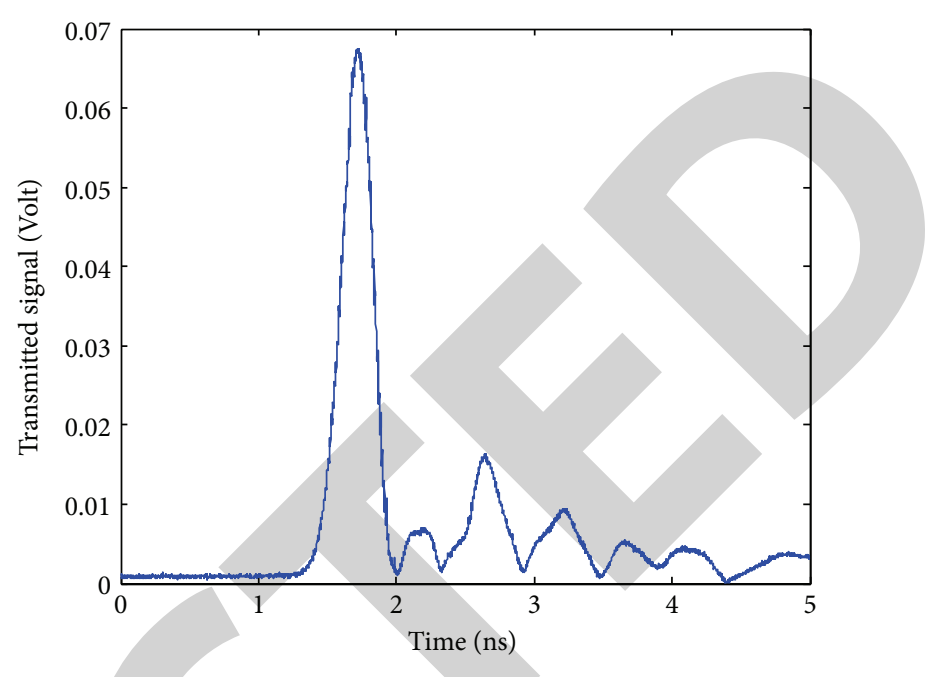

Figure 1: Transmitted signal of a 300-picosecond Gaussian pulse shape.

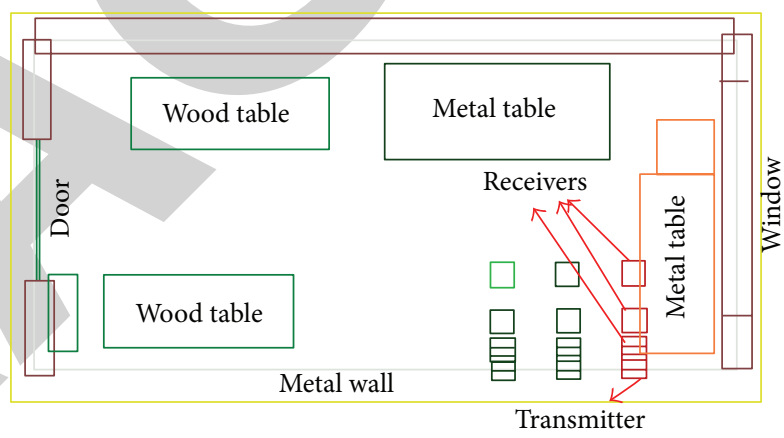

FIGURE 2: The indoor environment considered in our experiment.

where $I_{k l}(t)$ and $Q_{k l}(t)$ are, respectively, the inphase and quadrature component processes, $X_{k l}^{I}(t)$ and $X_{k l}^{\mathrm{Q}}(t)$ are, respectively, the state vectors of the inphase and quadrature components, $\left\{W_{k l}^{I}(t)\right\}_{t \geq 0}$ and $\left\{W_{k l}^{\mathrm{Q}}(t)\right\}_{t \geq 0}$ are two independent standard Brownian motions which correspond to the inphase and quadrature components, respectively, and $A_{k l}^{I}(t), A_{k l}^{\mathrm{Q}}(t)$, $B_{k l}^{I}(t), B_{k l}^{\mathrm{Q}}(t), H_{k l}^{I}(t)$, and $H_{k l}^{\mathrm{Q}}(t)$ are matrices of appropriate dimensions. Note that $I_{k l}(t)$ and $Q_{k l}(t)$ are two independent Gaussian processes with zero-mean and equal variances; that is, $\operatorname{Var}\left(I_{k l}(t)\right)=\operatorname{Var}\left(Q_{k l}(t)\right)=\operatorname{Var}\left(N_{k l}(t)\right)$, where $N_{k l}(t)$ is either $I_{k l}(t)$ or $Q_{k l}(t)$ [13]. They are related to the tap weight $a_{k l}(t, \tau)$ and phase $\phi_{k l}(t, \tau)$ by the expressions $a_{k l}(t, \tau)=$ $\sqrt{I_{k l}^{2}(t, \tau)+Q_{k l}^{2}(t, \tau)}$ and $\phi_{k l}(t, \tau)=\arctan \left(Q_{k l}(t, \tau) / I_{k l}(t, \tau)\right)$, respectively. In the next section, the stochastic narrowband and UWB indoor channel models are developed.

\section{Stochastic State-Space Models for UWB Indoor Wireless Channels}

In narrowband systems, the equivalent indoor complex baseband representation consists of Rayleigh-distributed amplitude and uniformly distributed phase [20]. Thus, the 


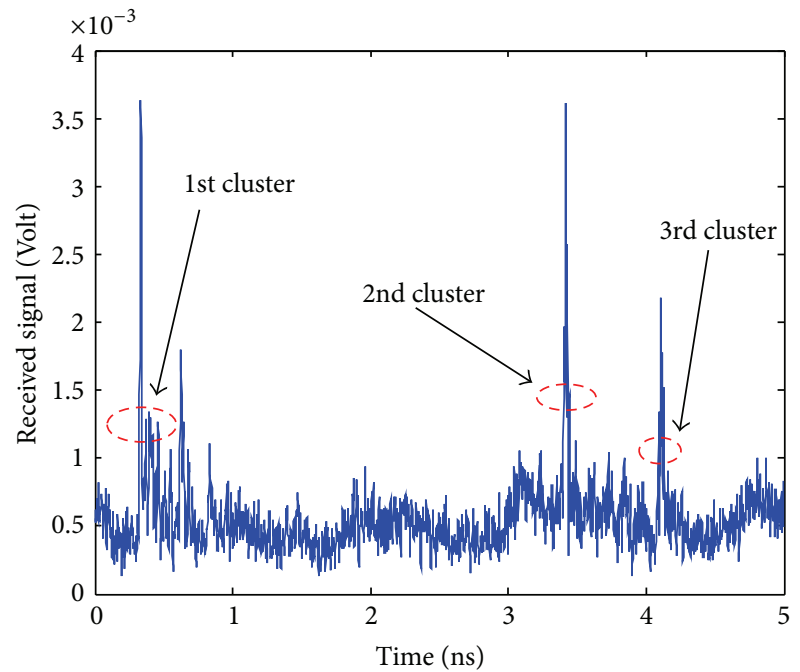

FIGURE 3: The measured UWB received signal.

stochastic narrowband indoor state-space channel model can be represented as

$$
\begin{aligned}
d X_{k l}(t)= & A_{k l}(t) X_{k l}(t) d t+B_{k l}(t) d W_{k l}(t), \\
y(t)=\sum_{l=1}^{L} \sum_{k=1}^{K_{l}} & \sqrt{\left(C_{k l}^{I}(t) X_{k l}(t)\right)^{2}+\left(C_{k l}^{\mathrm{Q}}(t) X_{k l}(t)\right)^{2}} \\
& \times e^{j \arctan \left(C_{k l}^{\mathrm{Q}}(t) X_{k l}(t) / C_{k l}^{I}(t) X_{k l}(t)\right)} \\
& \times s\left(t-T_{l}(t)-\tau_{k l}(t)\right)+D(t) v(t),
\end{aligned}
$$

where

$$
\begin{gathered}
X_{k l}(t)=\left[\begin{array}{ll}
X_{k l}^{I}(t)^{T} & X_{k l}^{\mathrm{Q}}(t)^{T}
\end{array}\right]^{T}, \\
W_{k l}(t)=\left[\begin{array}{ll}
W_{k l}^{I}(t)^{T} & W_{k l}^{\mathrm{Q}}(t)^{T}
\end{array}\right]^{T}, \\
A_{k l}(t)=\left[\begin{array}{cc}
A_{k l}^{I}(t) & 0 \\
0 & A_{k l}^{\mathrm{Q}}(t)
\end{array}\right], \\
B_{k l}(t)=\left[\begin{array}{cc}
B_{k l}^{I}(t) & 0 \\
0 & B_{k l}^{\mathrm{Q}}(t)
\end{array}\right], \\
C_{k l}^{I}(t)=\left[\begin{array}{ll}
H_{k l}^{I}(t) & 0
\end{array}\right], \quad C_{k l}^{\mathrm{Q}}(t)=\left[\begin{array}{l}
\left.0 H_{k l}^{\mathrm{Q}}(t)\right], \\
I_{k l}(t, \tau)=C_{k l}^{I}(t) X_{k l}(t), \quad Q_{k l}(t, \tau)=C_{k l}^{\mathrm{Q}}(t) X_{k l}(t),
\end{array}\right.
\end{gathered}
$$

where $v(t)$ is the measurement noise which is assumed to be Gaussian with zero-mean and unit variance and the tap weight process $a_{k l}(t, \tau)=$ $\sqrt{\left(C_{k l}^{I}(t) X_{k l}(t)\right)^{2}+\left(C_{k l}^{Q}(t) X_{k l}(t)\right)^{2}}$ and the phase process $\phi_{k l}(t, \tau)=\arctan \left(C_{k l}^{\mathrm{Q}}(t) X_{k l}(t) / C_{k l}^{I}(t) X_{k l}(t)\right)$ are independent Rayleigh- and uniform-distributed random processes, respectively. In this case, the tap weight process $a_{k l}(t, \tau)$ has the following statistics [25]:

$$
\begin{aligned}
E\left\{a_{k l}(t, \tau)\right\} & =\sqrt{\frac{\pi}{2} \operatorname{Var}\left(N_{k l}(t)\right)}, \\
E\left\{\left(a_{k l}(t, \tau)\right)^{p}\right\} & =\left(2 \operatorname{Var}\left(N_{k l}(t)\right)\right)^{p / 2} \Gamma\left(1+\frac{1}{2} p\right), \\
\operatorname{Var}\left\{a_{k l}(t, \tau)\right\} & =\left(2-\frac{\pi}{2}\right) \operatorname{Var}\left(N_{k l}(t)\right),
\end{aligned}
$$

where $\Gamma(p)$ is the gamma function.

In UWB systems, as mentioned earlier, the most common amplitude distribution of the received signal is Nakagami distribution [6-8]. Its probability density function is given by [26]

$$
f(x)=\frac{2}{\Gamma(m)}\left(\frac{m}{\Omega}\right)^{m} x^{2 m-1} \exp \left(-\frac{m}{\Omega} x^{2}\right),
$$

where $m \geq 0.5$ is the shape parameter, $\Gamma(m)$ is the Gamma function, and $\Omega$ controls the spread of distribution. The $m$ parameter is often modeled as a random variable [6]. For integer value of $m$, the distribution describes $m$ orthogonal independent Rayleigh-distributed random variables. That is, for $M$ Rayleigh-distributed random variables $Z_{i}$, the probability density function of random variable $Y$, defined as $Y=\sqrt{\sum_{i=1}^{M} Z_{i}^{2}}$, is given by a Nakagami distribution with parameter $m=M$ [27].

Since multiple orthogonal independent Rayleigh-distributed random variables can generate Nakagami distribution, the stochastic UWB indoor state-space channel model can be represented by

$$
\begin{aligned}
& d X_{i k l}(t)=A_{i k l}(t) X_{i k l}(t) d t+B_{i k l}(t) d W_{i k l}(t), \\
& y(t)=\sum_{l=1}^{L} \sum_{k=1}^{K_{l}} \sqrt{\sum_{i=1}^{M_{k l}}\left(C_{i k l}^{I}(t) X_{i k l}(t)\right)^{2}+\left(C_{i k l}^{Q}(t) X_{i k l}(t)\right)^{2}} \\
& \times e^{j \arctan \left(C_{i k l}^{Q}(t) X_{i k l}(t) / C_{i k l}^{I}(t) X_{i k l}(t)\right)} \\
& \times s\left(t-T_{l}(t)-\tau_{k l}(t)\right)+D(t) v(t),
\end{aligned}
$$

where

$$
\begin{aligned}
& X_{i k l}(t)=\left[X_{i k l}^{I}(t)^{T} X_{i k l}^{Q}(t)^{T}\right]^{T} \text {, } \\
& W_{i k l}(t)=\left[W_{i k l}^{I}(t)^{T} W_{i k l}^{\mathrm{Q}}(t)^{T}\right]^{T} \text {, } \\
& A_{i k l}(t)=\left[\begin{array}{cc}
A_{i k l}^{I}(t) & 0 \\
0 & A_{i k l}^{Q}(t)
\end{array}\right], \\
& B_{i k l}(t)=\left[\begin{array}{cc}
B_{i k l}^{I}(t) & 0 \\
0 & B_{i k l}^{Q}(t)
\end{array}\right], \\
& C_{i k l}^{I}(t)=\left[\begin{array}{ll}
H_{i k l}^{I}(t) & 0
\end{array}\right], \quad C_{i k l}^{\mathrm{Q}}(t)=\left[\begin{array}{ll}
0 H_{i k l}^{\mathrm{Q}}(t)
\end{array}\right], \\
& I_{i k l}(t, \tau)=C_{i k l}^{I}(t) X_{i k l}(t), \quad Q_{i k l}(t, \tau)=C_{i k l}^{Q}(t) X_{i k l}(t) \text {, }
\end{aligned}
$$




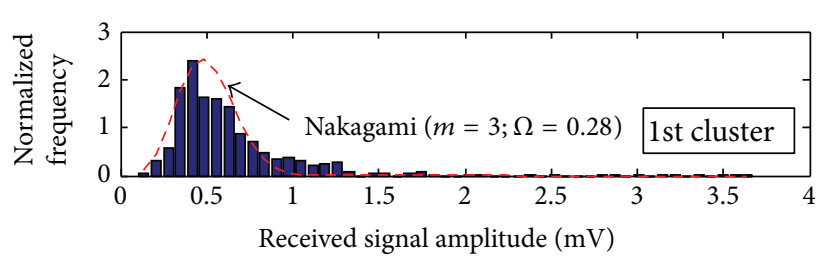

(a)

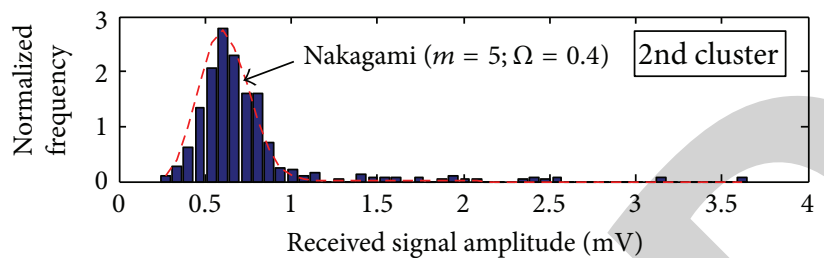

(b)

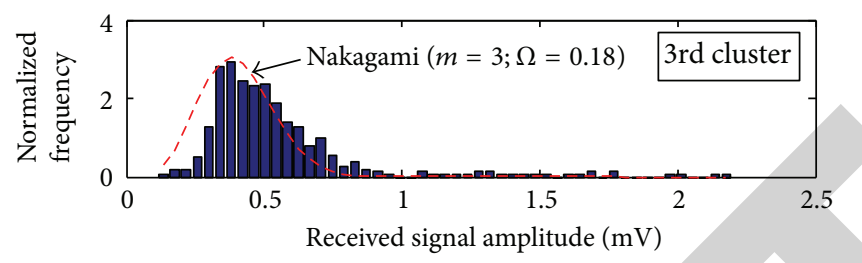

Measurements Nakagami distribution

(c)

FIGURE 4: Histogram of measurement data for the dominant paths within the three clusters that are best fit to Nakagami distributions.

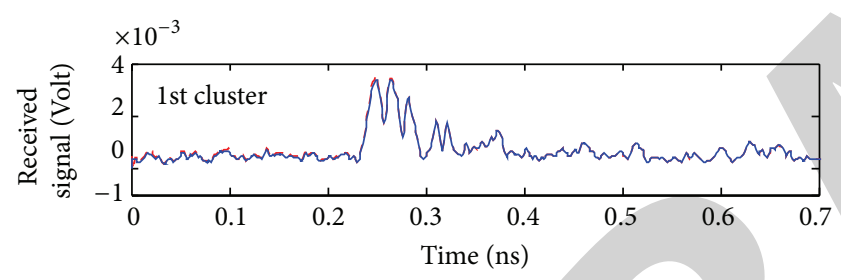

(a)

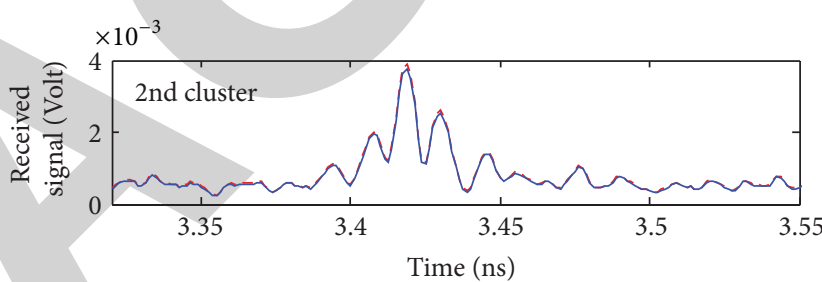

(b)

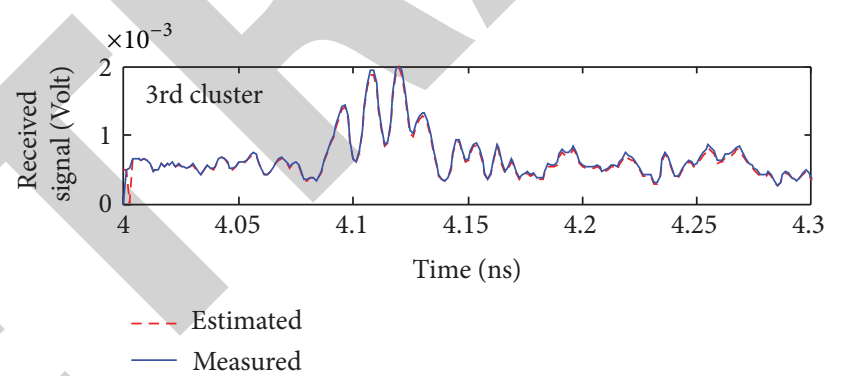

(c)

FIGURE 5: Measured and estimated received signals using the EM algorithm combined with the EKF for the 1st, 2nd, and 3rd clusters.

where $L$ is the total number of clusters, $K_{l}$ is the total number of resolvable delay bins (paths), and $M_{k l}$ is the number of nonresolvable delay bins within the resolvable delay bin $k$ in cluster $l$. In this case, the tap weight process $a_{k l}(t, \tau)$ has the following statistics [25]:

$$
\begin{gathered}
E\left\{a_{k l}(t, \tau)\right\}=\sqrt{2 \operatorname{Var}\left(N_{k l}(t)\right)} \frac{\Gamma\left((1 / 2)\left(2 M_{k l}+1\right)\right)}{\Gamma\left(M_{k l}\right)}, \\
E\left\{\left(a_{k l}(t, \tau)\right)^{p}\right\}=\left(2 \operatorname{Var}\left(N_{k l}(t)\right)\right)^{p / 2} \frac{\Gamma\left((1 / 2)\left(2 M_{k l}+p\right)\right)}{\Gamma\left(M_{k l}\right)}, \quad p \geq 0, \\
\Omega_{k l}=2 \operatorname{Var}\left(N_{k l}(t)\right) \frac{\Gamma\left(M_{k l}+1\right)}{\Gamma\left(M_{k l}\right)} .
\end{gathered}
$$

It can be noticed in (30) that the received signal measurement, $y(t)$, is a nonlinear function of the state variables of the model. The UWB channel parameters are estimated using the EM algorithm, and the inphase and quadrature components are estimated directly from received signal measurements using the EKF. A filter-based EM algorithm together with the EKF is employed to estimate the channel model parameters and states in (30). These filters use only the first- and second-order statistics and are also recursive and therefore can be implemented online. These algorithms have been recently utilized in [11-13] to estimate the channel parameters and states in narrowband environments, and therefore the formulations of these algorithms are not presented in this paper. Experimental results demonstrating the applicability of these algorithms in UWB indoor environments are discussed in the next section. 


\section{Experimental Setup and Numerical Results}

A simple Gaussian pulse with clean pulse shape and narrow pulse width is chosen as UWB source signal. The experimental setup is similar to the one in [28]. It comprises a 300 picosecond Gaussian pulse (see Figure 1) that modulates a carrier signal centered at $8 \mathrm{GHz}$ and is transmitted through an omnidirectional UWB antenna. Multiple directional Vivaldi subarray receiving antennas are located at distinct locations in an indoor environment to receive the modulated pulse signal. Each received modulated Gaussian pulse is amplified through a low noise amplifier (LNA) and then stored in a multichannel Tektronix TDS8200 sampling oscilloscope.

The transmitter is located next to a metal wall. The receiving antenna is placed $10 \mathrm{~cm}$ away from the transmitter and then moved along the same direction away from the transmitter for $20 \mathrm{~cm}, 50 \mathrm{~cm}, 1 \mathrm{~m}$, and $2 \mathrm{~m}$. This scenario is demonstrated in Figure 2. In this environment, the transmitted signal suffers from reflection from the metal wall, ceiling, or floor or is scattered by the corner of the metal table, and then it reaches the receiver. Figure 3 shows the measured received signal which consists of three clusters. The measurement data are amplified by $30 \mathrm{~dB}$ and it can be seen in Figure 4 that the dominant path in each cluster is best fit to Nakagami distribution with different parameters. For example, the dominant path in the first cluster is best fit to Nakagami distribution with parameters $m=3$ and $\Omega=$ 0.28. This indicates that there are three nonresolvable delay bins within this path (i.e., $M_{11}=3$ ). Figure 5 shows the measured and estimated received signals using the EM algorithm together with the EKF for the three clusters using a 4 th-order model. It can be noticed that the UWB received signal has been estimated with very high accuracy and it takes little iteration for the estimation algorithm to converge. At a certain time instant, the system parameters for the dominant path in the 1st cluster, which consists of three nonresolvable delay bins, are estimated as follows:

$$
\begin{aligned}
\widehat{A}_{111} & =\left[\begin{array}{cccc}
0 & 1 & 0 & 0 \\
0.1928 & 0.0556 & 0 & 0 \\
0 & 0 & 0 & 1 \\
0 & 0 & 0.1484 & -0.0966
\end{array}\right], \\
\widehat{A}_{211} & =\left[\begin{array}{cccc}
0 & 1 & 0 & 0 \\
0.2917 & 0.0556 & 0 & 0 \\
0 & 0 & 0 & 1 \\
0 & 0 & 0.3563 & -0.0682
\end{array}\right], \\
\widehat{A}_{311} & =\left[\begin{array}{cccc}
0 & 1 & 0 & 0 \\
0.2014 & -0.0586 & 0 & 0 \\
0 & 0 & 0 & 1 \\
0 & 0 & 0.1881 & -0.2669
\end{array}\right], \\
\widehat{B}_{111}^{2} & =\left[\begin{array}{llll}
0.1699 & 0.2451 & 0.0122 & 0.0009 \\
0.0004 & 0.0961 & 0.0287 & -0.0004 \\
0.0122 & 0.0087 & 0.1628 & 0.0213 \\
0.0077 & 0.0254 & 0.2959 & -0.0066
\end{array}\right],
\end{aligned}
$$

$$
\begin{aligned}
& \begin{aligned}
\widehat{B}_{211}^{2}= & {\left[\begin{array}{cccc}
0.0006 & 0.0182 & 0.1429 & 0.0077 \\
0.0043 & -0.0256 & 0.0076 & -0.2597 \\
0.0795 & 0.0056 & 0.0043 & 0.0218 \\
-0.0177 & 0.0192 & 0.1541 & -0.1632
\end{array}\right] } \\
\widehat{B}_{311}^{2}= & {\left[\begin{array}{cccc}
0.2561 & 0.0013 & 0.3310 & 0.0069 \\
0.0129 & 0.0213 & -0.0107 & 0.0921 \\
0.0044 & 0.0015 & 0.0824 & 0.0076 \\
0.1541 & 0.1429 & 0.1632 & -0.3334
\end{array}\right] } \\
& \widehat{C}_{111}^{I}=\widehat{C}_{211}^{I}=\widehat{C}_{311}^{I}=\left[\begin{array}{llll}
1 & 0 & 0 & 0
\end{array}\right]
\end{aligned} \\
& \widehat{C}_{111}^{\mathrm{Q}}=\widehat{C}_{211}^{\mathrm{Q}}=\widehat{C}_{311}^{\mathrm{Q}}=\left[\begin{array}{llll}
0 & 0 & 1 & 0
\end{array}\right] \text {, } \\
& \widehat{D}^{2}=[0.8765] \text {. }
\end{aligned}
$$

Notice that the EM algorithm estimates $\widehat{B}^{2}$ and $\widehat{D}^{2}$ instead of $\widehat{B}$ and $\widehat{D}$. Also note that the parameters $L, K_{l}, M_{k l}, T_{l}$, and $\tau_{k l}$ are assumed known through the estimation process. In fact, the parameters $L, K_{l}$, and $M_{k l}$ can be estimated by various estimation algorithms such as minimum description length [29], multiple hypothesis testing [30], exponential fitting test [31], and linear piecewise variation [32]. And a sounding device is usually dedicated to estimate the time delay of each discrete path (i.e., $T_{l}$ and $\tau_{k l}$ ) such as the rake receiver [33].

\section{Conclusion}

This paper describes a general scheme for extracting mathematical UWB indoor channel models from noisy received signal measurements. The UWB channel models are represented in stochastic state-space form, in which its system output produces Nakagami-distributed received signal strength and it is shown to approximate the general TV IR of the channel as close as desired. Experimental results indicate that the measured data can be regenerated with high accuracy.

\section{Acknowledgments}

This paper has been authored by employees of UT-Battelle, LLC, under Contract no. DE-AC05-00OR22725 with the US Department of Energy. Also, this work was supported in part by NSF Grant no. CMMI-1334094.

\section{References}

[1] Federal Communications Commission, "First report 02-48," 2002.

[2] M. Z. Win and R. A. Scholtz, "Impulse radio: how it works," IEEE Communications Letters, vol. 2, no. 2, pp. 36-38, 1998.

[3] A. F. Molisch, Wireless Communications, IEEE Press/Wiley, New York, NY, USA, 2005.

[4] R. C. Qiu, "A generalized time domain multipath channel and its application in ultra-wideband (UWB) wireless optimal receiver-part III: system performance analysis," IEEE Transactions on Wireless Communications, vol. 5, no. 10, pp. 2685-2695, 2006. 
[5] A. F. Molisch, "Ultrawideband propagation channels-theory, measurement, and modeling," IEEE Transactions on Vehicular Technology, vol. 54, no. 5, pp. 1528-1545, 2005.

[6] D. Cassioli, M. Z. Win, and A. F. Molisch, "The ultra-wide bandwidth indoor channel: from statistical model to simulations," IEEE Journal on Selected Areas in Communications, vol. 20, no. 6, pp. 1247-1257, 2002.

[7] A. F. Molisch, D. Cassioli, C. C. Chong et al., "A comprehensive standardized model for ultrawideband propagation channels," IEEE Transactions on Antennas and Propagation, vol. 54, no. 11, pp. 3151-3166, 2006.

[8] J. Kunisch and J. Pamp, "Measurement results and modeling aspects for the UWB radio channel," in Proceedings of the IEEE Ultra Wide-Band Systems and Technologies (UWBST '02), pp. 19-23, 2002.

[9] J. Karedal, S. Wyne, P. Almers, F. Tufvesson, and A. F. Molisch, "Statistical analysis of the UWB channel in an industrial environment," in Proceedings of the 60th IEEE Vehicular Technology Conference (VTC '04), vol. 1, pp. 81-85, September 2004.

[10] W. Wilson, Linear System Theory, Prentice Hall, 1996.

[11] M. M. Olama, K. K. Jaladhi, S. M. Djouadi, and C. D. Charalambous, "Recursive estimation and identification of timevarying long-term fading channels," Research Letters in Signal Processing, vol. 2007, Article ID 17206, 5 pages, 2007.

[12] M. M. Olama, L. Yanyan, S. M. Djouadi, and C. D. Charalambous, "Time varying wireless channel modeling, estimation, identification, and power control from measurements," in Proceedings of the American Control Conference (ACC '07), pp. 3100-3105, July 2007.

[13] M. M. Olama, S. M. Djouadi, and C. D. Charalambous, "Stochastic differential equations for modeling, estimation and identification of mobile-to-mobile communication channels," IEEE Transactions on Wireless Communications, vol. 8, no. 4, pp. 1754-1763, 2009.

[14] T. R. Field and R. J. A. Tough, "Stochastic dynamics of the scattering amplitude generating k-distributed noise," Journal of Mathematical Physics, vol. 44, no. 11, pp. 5212-5223, 2003.

[15] K. E. Baddour and N. C. Beaulieu, "Autoregressive modeling for fading channel simulation," IEEE Transactions on Wireless Communications, vol. 4, no. 4, pp. 1650-1662, 2005.

[16] T. Feng, T. R. Field, and S. Haykin, "Stochastic differential equation theory applied to wireless channels," IEEE Transactions on Communications, vol. 55, no. 8, pp. 1478-1483, 2007.

[17] C. D. Charalambous, S. M. Djouadi, and S. Z. Denic, "Stochastic power control for wireless networks via SDEs: probabilistic QoS measures," IEEE Transactions on Information Theory, vol. 51, no. 12, pp. 4396-4401, 2005.

[18] M. M. Olama, S. M. Djouadi, and C. D. Charalambous, "Stochastic power control for time-varying long-term fading wireless networks," Eurasip Journal on Applied Signal Processing, vol. 2006, Article ID 89864, 2006.

[19] Y. Li, M. Olama, S. Djouadi, A. Fathy, and T. Kuruganti, "Stochastic UWB wireless channel modeling and estimation from received signal measurements," in Proceedings of the IEEE Radio and Wireless Symposium (RWS '09), pp. 195-198, January 2008.

[20] A. A. M. Saleh and R. A. Valenzuela, "A statistical model for indoor multipath propagation," IEEE Journal on Selected Areas in Communications, vol. 5, no. 2, pp. 128-137, 1987.

[21] S. Venkatesh, J. Ibrahim, and R. M. Buehrer, "A new 2-cluster Model for indoor UWB channel measurements," in Proceedings of the IEEE Antennas and Propagation Society International Symposium, pp. 946-949, June 2004.

[22] S. M. Djouadi, M. M. Olama, and Y. Li, “Optimal approximation of the impulse response of wireless channels by stochastic differential equations," IEEE Signal Processing Letters, vol. 15, pp. 896-899, 2008.

[23] R. Schatten, Norm Ideals of Completely Continuous Operators, Springer, Berlin, Germany, 1960.

[24] E. Schmidt, "Zur Theorie der linearen und nicht linearen Integralgleichungen," Mathematische Annalen, vol. 64, no. 2, pp. 161-174, 1907 (German).

[25] K. J. Astrom, Introduction to Stochastic Control Theory, Dover Publications, New York, NY, USA, 1970.

[26] M. D. Yacoub, J. E. V. Bautista, and L. Guerra de Rezende Guedes, "On higher order statistics of the Nakagami-m distribution," IEEE Transactions on Vehicular Technology, vol. 48, no. 3, pp. 790-794, 1999.

[27] N. C. Beaulieu and C. Cheng, "Efficient nakagami- $\mathscr{M}$ fading channel simulation," IEEE Transactions on Vehicular Technology, vol. 54, no. 2, pp. 413-424, 2005.

[28] C. Zhang, M. Kuhn, B. Merk, M. Mahfouz, and A. E. Fathy, "Development of an UWB indoor 3D positioning radar with millimeter accuracy," in Proceedings of the IEEE MTT-S International Microwave Symposium Digest, pp. 106-109, June 2006.

[29] E. Fishler and H. V. Poor, "Estimation of the number of sources in unbalanced arrays via information theoretic criteria," IEEE Transactions on Signal Processing, vol. 53, no. 9, pp. 3543-3553, 2005.

[30] P.-J. Chung, J. F. Böhme, A. O. Hero, and C. F. Mecklenbräuker, "Detection of the number of signals using a multiple hypothesis test," in Proceedings of the Sensor Array and Multichannel Signal Processing Workshop, pp. 221-224, July 2004.

[31] A. Quinlan, J.-P. Barbot, P. Larzabal, and M. Haardt, "Model order selection for short data: an Exponential Fitting Test (EFT)," Eurasip Journal on Advances in Signal Processing, vol. 2007, Article ID 71953, 11 pages, 2007.

[32] E. Radoi and A. Quinquis, "A new method for estimating the number of harmonic components in noise with application in high resolution radar," Eurasip Journal on Applied Signal Processing, vol. 2004, no. 8, pp. 1177-1188, 2004.

[33] B. Sklar, Digital Communications: Fundamentals and Applications, Prentice Hall, 2nd edition, 2001. 